\title{
STRING PICTURE OF GAUGE FIELDS
}

\author{
JACEK PAWEŁCZYK \\ Institute of Theoretical Physics, Warsaw University \\ Hoża 69, 00-681 Warsaw, Poland \\ E-mail: Jacek.Pawelczyk@fuw.edu.pl
}

\begin{abstract}
The article reviews attempts to formulate the theory of gauge fields in terms of a string theory.

1. Introduction. One of the major problems of contemporary physics of fundamental interactions is the non-perturbative formulation of the theory of strong interactions. Strong interactions are described by $S U(3)$ gauge theory coupled to fermions. It seems that the exact solution of such a theory is impossible. In order to simplify it one considers the limit $N \rightarrow \infty$ for $S U(N)$ theory and discard fermions at first approximation. It is strongly believed that in this limit the dynamics of gauge fields can be described in terms of a string theory $[13,14]$. The idea was supported in $[2,3,8-10]$ for the theory formulated in a 2-dimensional space-time. The string picture for such a gauge theory has been proposed in several papers $[5,15,16,21]$ and will hopefully help to formulate the physical theory of gauge fields in 4-dimensional spaces.

In Sections 2 and 3 we review string picture of two-dimensional gauge fields $\left(\mathrm{YM}_{2}\right)$. Physics of these models reveals a connection with interesting mathematical structures which will be discussed in the course of this paper. It appears that the limit $N \rightarrow \infty$ of $\mathrm{YM}_{2}$ is closely related to the moduli space of branched coverings and Hurwitz space. In the last section we shall discuss the possible role of the self-intersection number of a surface immersed in $\mathbb{R}^{4}$ in the formulation of a string theory of 4-dimensional gauge fields.
\end{abstract}

In the next section we are going to make a string interpretation of a formula for the partition function of $\mathrm{YM}_{2}[19,22]$ :

$$
Z=\sum_{R \in \operatorname{Rep}(S U(N))}(\operatorname{dim} R)^{2-2 G} e^{-\frac{A}{2 N} C_{2}(R)},
$$

1991 Mathematics Subject Classification: Primary 81T13; Secondary 81T30.

Research supported by KBN grant.

The paper is in final form and no version of it will be published elsewhere. 
where the sum is over all irreducible representations of the gauge group, i.e. $S U(N)$ in our case, $M_{G}$ is a Riemann surface of the genus $G>1$ and $A$ denote the volume of $M_{G}$. Before we go to the interpretation we must specify what we mean by a string theory. At heuristic level a string theory is a theory of maps $X$ from 2-dimensional surfaces (called world-sheets) $\Sigma$ to a space-time $M_{G}$. Here we can restrict our considerations to closed, oriented world-sheets (Riemann surfaces) of the genus $g$ thus sometimes we write $\Sigma_{g}$ instead of $\Sigma$. The theory is defined through a 'functional integral' which give a partition function of the theory. The $1 / N$ expansion of gauge theories $[13,14]$ suggests that the partition function should have the following form:

$$
Z \propto \sum_{g=0}^{\infty}\left(\frac{1}{N}\right)^{2 g-2} \sum_{X: \Sigma_{g} \rightarrow M_{G}} e^{-S[X]},
$$

As we see (2) is a series in $1 / N$ and the powers of $1 / N$ have a topological form, i.e. they are the Euler characteristics of $\Sigma$. The functional $S[X]$ should be specified in the course of unveiling connection between gauge theories and a string theory. The whole recent progress in building this connection lies in the fact that indeed the functional integral of $\mathrm{YM}_{2}$ had been rewritten in the above form with more or less clear interpretation of the $\operatorname{sum} \sum_{X: \Sigma_{g} \rightarrow M_{G}}$ and definite $S[X]$.

2. String interpretation of the $1 / N$ expansion. In this section we shall discuss the $1 / N$ expansion of the result (1) and its string picture. The idea of the calculation is to use Schur-Weyl duality to translate $S U(N)$ representations with $n$ boxes in Young tableaux into $S_{n}$ representation theory, and interpret the latter, geometrically, as data defining a branched covering $\Sigma \rightarrow M_{G}$ of degree $n$.

Definition. A continuous map $X: \Sigma \rightarrow M_{G}$ is a branched covering if any point $p \in M_{G}$ has a neighborhood $U \subset M_{G}$, such that the inverse image $X^{-1}(U)$ is a union of disjoint open sets (enumerated by $k$ ) on each of which $X$ is topologically equivalent to the complex map $z \mapsto z^{n_{k}(p)}$ for some $n_{k}(p) \in Z_{+}$.

Moreover we define the branched locus $\mathcal{S}$ and the branching number $B$.

Definition. $\mathcal{S}=\left\{p \in M_{G}: n_{k}(p)>1\right\}$.

Definition. $B=\sum_{p \in \mathcal{S}, k}\left[n_{k}(p)-1\right]$.

Some details of the construction will be given below. For the full presentation we refer the reader to the original literature [5,8-10].

Let us rewrite the sum (1) as

$$
\sum_{R \in \operatorname{Rep}(S U(N))}=\sum_{n \geq 0} \sum_{Y \in \mathcal{Y}_{n}^{(N)}},
$$

where $\mathcal{Y}_{n}^{(N)}$ denotes a set of Young tableaux of $S U(N)$ with $n$ boxes. We must decide what are the leading terms in the $N \rightarrow \infty$ expansion. Getting the correct asymptotic expansion requires isolating all representations which have $\frac{C_{2}(R)}{N} \sim O\left(N^{0}\right)$, and making sure their contributions appear after a finite number of terms. In [8-10] it was argued that the most general representations which satisfy $\frac{C_{2}(R)}{N} \sim O\left(N^{0}\right)$ are the 'coupled 
representations'. The latter are defined as those which appear in the tensor product of $T$ and $\bar{T}^{\prime}$ under the condition that the number of rows in the Young tableaux of $T^{\prime}$ and $T$ divided by $N$ tends to zero for $N \rightarrow \infty$. Below we shall describe only the so-called one 'chiral sector' of the model. It is defined by dropping the constraint on the number of rows in $(3)$.

In order to accomplish the idea we need to express the second Casimir $C_{2}(R)$ and the dimension of the representation $\operatorname{dim}(R)$ in the symmetric group language. Then,

$$
C_{2}(R(Y))=n N+2 \frac{\chi_{r(Y)}\left(T_{2, n}\right)}{d_{r(Y)}}-\frac{n^{2}}{N},
$$

where $T_{2, n}$ denotes the conjugacy class of permutations containing a single cycle of length 2 and $n-2$ cycles of length $1, d_{r(Y)}$ is the dimension of the representation $r$ of the symmetric group associated with the Young tableau $Y$. Another important formula is the expression for the powers of dimensions of $S U(N)$ representations in terms of characters of the symmetric group:

$$
\operatorname{dim} R(Y)=\frac{N^{n}}{n !} \chi_{r(Y)}\left(\Omega_{n}\right)=\frac{1}{n !} \sum_{\sigma \in S_{n}} N^{K_{\sigma}} \chi_{r(Y)}(\sigma)
$$

where $\Omega_{n}=\sum_{\sigma \in S_{n}}\left(\frac{1}{N}\right)^{n-K_{\sigma}} \sigma$ and $K_{\sigma}$ is the number of cycles in the cycle decomposition of $\sigma$. With the help of (4)-(5) and a few identities among characters of $S_{n}$ we can perform the $N \rightarrow \infty$ expansion and get the following result for the partition function of the one 'chiral sector' of the model:

$Z^{+}(A, p, N)=1+\sum_{n=1, i, t, h=0}^{\infty} e^{-n A / 2}(-1)^{i} \frac{(A)^{i+t+h}}{i ! t ! h !}\left(\frac{1}{N}\right)^{n(2 G-2)+2 h+i+2 t}\left(\frac{n}{2}\right)^{h}\left(\frac{n(n-1)}{2}\right)^{t}$

$$
\sum_{p_{1}, \ldots, p_{i} \in T_{2, n}} \sum_{s_{1}, t_{1}, \ldots, s_{G}, t_{G} \in S_{n}}\left[\frac{1}{n !} \delta\left(p_{1} \cdots p_{i} \Omega_{n}^{2-2 G} \prod_{j=1}^{G} s_{j} t_{j} s_{j}^{-1} t_{j}^{-1}\right)\right] .
$$

Acting on an element of the group algebra, the delta function evaluates the element (regarded as a function on the group) at the identity of $S_{n}$.

Below we present geometrical interpretation of the $A$-dependence in (6). There we have a sum over 4 positive integers, $n, i, t, h$. We will associate geometrical pictures with the $A$-dependence coming from these sums. These pictures are meant to be heuristic and sometimes may be misleading. First we notice that for $t=h=0$ the power of $1 / N$ is the Riemann-Hurwitz relation:

$$
2 g-2=n(2 G-2)+i
$$

for branched coverings. Thus we interpret $n$ as the number of sheets of the cover, i.e. degree of the branched covering. Then $e^{-n A / 2}$ must come from $e^{-S[X]}$ where $S[X]$ is half of the area functional. Due to the $(A)^{i+t+h}$ factor we associate $(x=i, t, h)$ with numbers of marked point of the type specified below. The $\frac{1}{x !}(x=i, t, h)$ is understood as a symmetry factor due to the marked points of a given type being indistinguishable. The interpretation of $(i, t, h)$ goes as follows. The sum over $i$ is counting branched covers with $i$ points being simple branch points, with weight equal to the inverse of the order of the automorphism group of the cover. The sum over $t$ is interpreted as a sum over infinitesimally thin 
tubes connecting two sheets. The tubes result in a partial compactification of the space of branched coverings [5]. The factor $\frac{n(n-1)}{2}$ is the number of ways of choosing which pair of sheets is being connected by the tube. Contribution with $h>0$ represent maps for which a handle has been mapped to a point in $M_{G}$. For $t \neq 0, h \neq 0$ the equality $2 g-2=n(2 G-2)+i+2 h+2 t$ should be treated as the generalized Riemann-Hurwitz relation.

In this way we have reformulated the partition function of $\mathrm{YM}_{2}$ in terms of a string theory. The big challenge is to calculate this partition function as a functional integral over string variables. The purpose has not been achieved although there exist partially successful proposals $[5,15,16,21]$.

3. Hurwitz spaces and $\mathbf{Y M}_{2}$ partition function. In this section we shall show that the partition function (6) is closely related to the Euler characteristic of the Hurwitz space [5]. The relation holds only for $G>1$ thus here we shall limit our considerations to this case. The Hurwitz space of branched coverings is described in $[6,11]$.

Definition. Two branched coverings $X_{1}$ and $X_{2}$ are said to be equivalent if there exists a homeomorphism $\phi: \Sigma_{W} \rightarrow \Sigma_{W}$ such that $X_{1} \circ \phi=X_{2}$.

Definition. Let $H(n, B, G ; \mathcal{S})$ be the set of equivalence classes of branched coverings of $M_{G}$, with degree $n$, branch locus $\mathcal{S}$, where $\mathcal{S}$ is a set of distinct points on a surface $M_{G}$ and branching number $B$. The union of $H(n, B, G ; \mathcal{S})$ over sets $\mathcal{S}$ with $L$ elements is the Hurwitz space $H(n, B, G, L)$ of equivalence classes of branched coverings of $M_{G}$ with degree $n$, branching number $B$ and $L$ branch points.

Moreover we introduce the configuration space of ordered $L$-tuples of distinct points on $M_{G}$, that is

$$
C_{L}(G)=\left\{\left(z_{1}, \ldots, z_{L}\right) \in M_{G}^{L}: z_{i} \in M_{G}, z_{i} \neq z_{j} \text { for } i \neq j\right\} .
$$

The permutation group $S_{L}$ acts naturally on $C_{L}$ and we denote the quotient $\mathcal{C}_{L}(G)=$ $C_{L}(G) / S_{L}$. There is a projection

$$
\pi: H(n, B, G, L) \rightarrow \mathcal{C}_{L}(G),
$$

which assigns to each branch covering its branch locus. This map can be made a covering map with the discrete fiber $H(n, B, G ; \mathcal{S})$ over $\mathcal{S} \in \mathcal{C}_{L}$.

$$
\begin{gathered}
H(n, B, G ; \mathcal{S}) \rightarrow \quad H(n, B, G, L) \\
\downarrow \\
\mathcal{C}_{L} .
\end{gathered}
$$

The lifting of closed curves in $\mathcal{C}_{L}$ will in general permute different elements of the fibers $H(n, B, G, \mathcal{S})$.

In the following we shall make a connection between the topology of Hurwitz space and $Y M_{2}$. We shall study the limit $A \rightarrow 0$ of the partition function. It appears that the limit is suitable for the formulation of the partition function in terms of a string theory (of a complicated structure) with the help of topological field theory [8-10,28]. We shall allude on this point in the end of this section. 
Thus we consider:

$$
Z^{+}(0, G, N)=1+\sum_{n=1}^{\infty} N^{n(2-2 G)} \sum_{s_{1}, t_{1}, \ldots, s_{G}, t_{G} \in S_{n}}\left[\frac{1}{n !} \delta\left(\Omega_{n}^{2-2 G} \prod_{j=1}^{G} s_{j} t_{j} s_{j}^{-1} t_{j}^{-1}\right)\right] .
$$

Our aim is to rewrite $Z^{+}$as a sum over equivalence classes of branched coverings and interpret the weights in terms of the Euler characters of the Hurwitz space. We present here few steps leading to this result. Expanding inverse powers of $\Omega_{n}$ in (11) we get

$$
\begin{aligned}
Z^{+}(0, G, N)=1+ & \sum_{n=1}^{\infty}\left(\frac{1}{N}\right)^{n(2 G-2)} \sum_{L=0}^{\infty} \sum_{s_{1}, t_{1}, \ldots, s_{G}, t_{G} \in S_{n}} \sum_{\sigma_{1} \neq 1 \ldots \sigma_{L} \neq 1 \in S_{n}} \\
& \left(\frac{1}{N}\right)^{B\left(\left\{\sigma_{i}\right\}\right)}\left[\frac{\chi\left(\mathcal{C}_{L}(\Sigma)\right)}{n !} \delta\left(\prod_{i=1}^{L} \sigma_{i} \prod_{j=1}^{G} s_{j} t_{j} s_{j}^{-1} t_{j}^{-1}\right)\right]
\end{aligned}
$$

where $\chi\left(\mathcal{C}_{L}(\Sigma)\right)$ is the Euler characteristic of $\mathcal{C}_{L}(\Sigma)$ given by

$$
\mathcal{C}_{L}(\Sigma)=\frac{\left(\chi_{G}\right)\left(\chi_{G}-1\right) \cdots\left(\chi_{G}-L+1\right)}{L !}
$$

and $B\left(\left\{\sigma_{i}\right\}\right)=\sum_{i=1}^{L}\left(n-K_{\sigma_{i}}\right)$. The latter is the branching number of the given branched covering associate with the data $\left\{\sigma_{i}\right\}$. The delta function divided by $n$ ! is in fact symmetry factor of the branched covering $X$, i.e. it is $1 / \mid$ Aut $X \mid$ where $\mid$ Aut $X \mid$ is the order of the group of automorphisms of $f$. Collecting terms of the same $B$ we get:

$$
Z^{+}(0, G, N)=1+\sum_{n=1}^{\infty} \sum_{B=0}^{\infty}\left(\frac{1}{N}\right)^{n(2 G-2)+B} \sum_{L=0}^{B} \chi\left(\mathcal{C}_{L}(\Sigma)\right) \sum_{X \in H(n, B, G ; \mathcal{S})} \frac{1}{\mid \text { Aut } X \mid}
$$

Having in mind the fact that the Hurwitz space is not a manifold but a orbifold we find out that the last two terms of formula (14) form the orbifold Euler characteristic [25] of the Hurwitz space. Thus we finally arrive at:

$$
Z^{+}(0, G, N)=1+\sum_{n=1}^{\infty} \sum_{B=0}^{\infty}\left(\frac{1}{N}\right)^{n(2 G-2)+B} \sum_{L=0}^{B} \chi_{\mathrm{orb}}(H(n, B, G ; L)) .
$$

This is the result claimed in the introduction.

With the help of the above result one can construct its field theory description ('functional integral'). The great advantage of branched coverings is that they allow the description of the Euler characteristic of the Hurwitz space in terms of topological field theory [28]. It is known that the topological field theories calculate the Euler number of the appropriate moduli space [1,18]. The moduli space, in our case, is the space of branched coverings. One can show that the latter is the same as the space of equivalent classes (under the action of $\operatorname{Diff}^{+}(\Sigma) \times \operatorname{Weyl}(\Sigma)$ ) of holomorphic maps $X: \Sigma \rightarrow M_{G}$ defined as

$$
d X \circ \epsilon=J \circ d X
$$

where $\epsilon, J$ are complex structures on $\Sigma$ and $M_{G}$, respectively. The whole construction of the appropriate topological field theory is quite involved and we do not have place to describe it here. Anyway it yields the well defined string theory in the sense of (2). 
4. Dynamical cancellation of folds. The results of the previous sections are definitely beautiful but the straightforward application of the topological field theory machinery yields quite involved (one could say 'ugly') theory. Moreover from physical point of view the description is unsatisfactory because it cannot be easily extended to 4-dimensional space-times (although see $[15,16]$ ). In this section we shall start a description of a different proposal [21]. This approach is not so developed as the previous one but offers an elegant picture of $N \rightarrow \infty$ limit of gauge theories. We shall concentrate here on one basic feature of the result (6), i.e. the property that the functional integral (understood as in (2)) suppresses folded configurations. In other words the functional integral is localized on a non-generic set of maps (16). In the beginning we recollect basic facts about generic surface to surface maps and their singularities $[7,26]$.

Let $X: \Sigma \rightarrow M_{G}$ denote a smooth map of a surfaces $\Sigma$ in a 2-dimensional manifold $M_{G}$ endowed with the metric $G_{\mu \nu}$ and $S_{1}(X)=\{p \in \Sigma: \operatorname{rank}(d X(p))=1\}$ its set of singular points. In a local coordinate patch the condition means that the matrix of the induced metric $g_{a b}=G_{\mu \nu} \partial_{a} X^{\mu} \partial_{b} X^{\nu}$ has one vanishing eigenvalue. For generic maps $S_{1}$ is a onedimensional submanifold of $\Sigma$. In particular, if $\Sigma$ is a closed surface (compact, connected, without boundary) then $S_{1}$ is a finite family of disjoint curves (loops) embedded in $\Sigma$. Next we define $S_{1,1}=\left\{p \in S_{1}: \operatorname{rank}\left(d\left(\left.X\right|_{S_{1}}(p)\right)=0\right\}\right.$, i.e. $S_{1,1}$ is a set of (generically disjoint) points at which eigenvector corresponding to zero eigenvalue is tangent to $S_{1}$. In a local coordinate system (simple) folds and cusps have the following form: folds, $\left(X^{1}, X^{2}\right)=\left(s^{2}, t\right)$; cusps, $\left(X^{1}, X^{2}\right)=\left(s t-s^{3}, t\right)$.

We know that generic maps do not contribute to the partition function (6). It means that any string representation of $\mathrm{YM}_{2}$ should have a built in mechanism of cancellation of these maps. One can localize the functional integral on a specific set of maps which do not contain folds. This is the idea of topological field theory and works $[5,15,16]$. Below we propose somewhat different model based of what might be called a dynamical cancellation of folds. We shall introduce a string model and show that for flat target space-times the model suppress generic, i.e. folded contributions. What about non-generic surface-tosurface maps? For flat space-times there are no non-singular maps and because the space of maps with folds and cusps is dense in the space of all smooth maps $[7,26]$ one can safely claim that these maps also do not contribute. Things get more complicated if the target space can be an arbitrary Riemann surface. Then there are maps without singularities and these cannot be simply discarded [20].

Let us introduce two additional (vertical) world-sheet fields: $\left(Y^{1}, Y^{2}\right) \in M_{v}\left(M_{v}\right.$ is a 'vertical' surface). The functional integral is over $\left(X^{1}, X^{2}, Y^{1}, Y^{2}\right) \in M_{G} \times M_{v}$ configurations. The latter can be viewed as lifts of $\left(X^{1}, X^{2}\right)$ configurations and it is known that generically they are immersions. The proposed string action is

$$
S[X, Y]=\mu \int_{M_{G}} d^{2} \sigma \sqrt{\operatorname{det}\left(g_{a b}\right)}+i \theta I[X, Y],
$$

where the first term is the area functional, $I$ is the self-intersection number of the surface immersed in the 4 -dimensional space $[12,17,24,27]$. The vertical coordinates enter the action only through $I$. The action (17) is invariant under all such homotopies of the vertical fields $\delta_{v} Y$ for which the map $\left(X, \delta_{v} Y\right)$ is regularly homotopic to $(X, Y)$. 
In the following we are going to classify topological sectors of the model. We say that two immersions are in the same topological sector if they can be connected by a $\delta_{v} Y$. In the following $X$ will denote a map $X: \Sigma \rightarrow M_{G}$ with folds and $(X, Y)$ its lift into the extended 4-dimensional space-time: $(X, Y): \Sigma \rightarrow M_{G} \times M_{v}$, where $M_{v}$ is an arbitrary orientable surface without boundary. We define the line bundle $K$ by $d X(K)=0$, where $d X$ is taken at points belonging to $S_{1}$ and the tangent map acts on the fiber over that point. Lifts of the fold must have non-degenerate 2-dimensional tangent space, hence must respect $d Y(K) \neq 0$. In this way the couple $(Y, d Y)$ defines a map (monomorphism) from $K$ to the tangent bundle of $M_{v}\left(T M_{v}\right)$. The set of connected components of such maps, define different topological sectors of lifts. The condition $d Y(K) \neq 0$ implies that this is the same as the set of connected components of maps from $K$ to one-dimensional sphere bundle over $M_{v}\left(S M_{v}\right)$. For one fold it is given by $\pi_{1}\left(S M_{v}\right)$. We conclude that $\pi_{1}\left(S M_{v}\right)$ classify lifts of one fold. Generalization for the case of a map with many folds is obvious, because lifts of folds are independent on each other. We may notice that the classification is analogous of that done by Smale for immersions of curves in Riemann surfaces [23].

As an application we take first $M_{G}=M_{v}=\mathbb{R}^{2}$. Then lifts of the $i$-th fold are classified by $\pi_{1}\left(\mathbb{R}^{2} \times S^{1}\right)=Z$. The integer $f_{i} \in \pi_{1}\left(\mathbb{R}^{2} \times S^{1}\right)$ is invariant under $\delta_{v} Y$ and is directly related to the self-intersection number $I$ of the lifted configuration. We can see it if we notice that both numbers are additive under connected sum. Let us associate a pair $(f, I)$ of numbers to a lift. If we glue it with the $\left(f^{\prime}, I^{\prime}\right)$ lift, we obtain the $\left(f+f^{\prime}, I+I^{\prime}\right)$ lift. Connected sum of $f_{1}$ copies of the $\left(-1,-I_{1}\right)$ lift with the $\left(f_{1}, 1\right)$ lift yields the $\left(0,1-f_{1} I_{1}\right)$ lift. If $K$ is trivial $f=0$ corresponds to $I=0$ [4]. Thus $1=f_{1} I_{1}$, so $I_{1}=f_{1}= \pm 1$ (the sign is undetermined). For non-trivial $K$ the intersection number may be shifted by an unessential (see below) integer. The same reasoning can be repeated for more complicated folds with several disconnected components and cusps. One assigns the numbers $f_{i} \in \pi_{1}\left(S^{1}\right)$ to $i$-th connected component of the set of folds. The numbers $\left\{f_{i}\right\}$ are invariant under the v-regular homotopy. The self-intersection number is then $I[f]=\sum_{\text {folds }} \pm f_{i}$. It is quite clear that topological sectors of the model are in one-to-one correspondence with sets $\left\{f_{i}\right\}[21]$.

Now we apply these results to the string theory on $M_{G}=\mathbb{R}^{2}$. We want to show that the originally folded configurations $\left(X^{1}, X^{2}\right)$ will cancel out from the partition function. Here one should discuss the construction of the functional integral measure of the theory. The heuristic construction of such a measure was given in [20] and we omit it here. The claim is that the functional integral is

$$
\int \mathcal{D} X^{1} \mathcal{D} X^{2} e^{-S[X]} F[X] \sum_{\left\{f_{i}\right\}} e^{i \theta I[f]},
$$

where the functional $F[X]$ is yet undefined (and unimportant for cancellation of folds). The sum over $f_{i}$ 's can be performed independently for each $i$ because $I[f]=\sum_{\text {folds }} \pm f_{i}$. For one fold we get

$$
\sum_{f \in Z} e^{ \pm i \theta f}=2 \pi \delta(\theta)
$$


Thus all folded configurations vanish from the path integral for non-zero $\theta$. All maps defining the partition function of the string theory necessarily have folds for the target space $\mathbb{R}^{2}$. Thus according to the above discussion it must vanish. This also holds for any average of any finite set of local operators. Thus the final conclusion of this part of the paper is that the model (17) is trivial for the $\mathbb{R}^{2}$ space-time.

The model discussed here contains one addition parameter compared to $\mathrm{YM}_{2}$ with semi-simple Lie group: it is the $\theta$ angle. In the following we shall claim that this $\theta$ is inherited from $\mathrm{YM}_{4}$. One can view (17) as a certain compactification of a 4-dimensional string. Thus $M_{v}$ should be a compact manifold without boundary. If a characteristic size of this space is small we expect that quantum fluctuations in the compactified directions are strongly suppressed. The case considered previously $M_{v}=\mathbb{R}^{2}$ is a variation of compactification on a torus. The standard compactification of $\mathrm{YM}_{4}$ on a 2-dimensional torus leads to additional 2-dimensional degrees of freedom: the adjoint matter. It is because the 4-dimensional gauge fields $A_{\mu}^{a}\left(x^{1}, \ldots, x^{4}\right)(\mu=1, \ldots, 4, a$ is the adjoint representation index) decompose into $\left(A_{\alpha}^{a}\left(x^{1}, x^{2}\right), A_{3}^{a}\left(x^{1}, x^{2}\right), A_{4}^{a}\left(x^{1}, x^{2}\right)\right)(a=1,2)$. From the point of view of the uncompactified 2-dimensional space-time $A_{3}^{a}, A_{4}^{a}$ are the matter fields. The appearance of the continuous $\theta$ parameter in (17) is natural in this case. It may correspond to the analogous angle in the QCD Lagrangian, because after compactification we have:

$$
\int d^{4} x \operatorname{tr}(F \tilde{F}) \rightarrow 4 \int d^{2} x \epsilon^{\alpha \beta} \operatorname{tr}\left(F_{\alpha \beta} A_{3} A_{4}\right)
$$

In order to get rid of these matter fields (and thus certain ambiguities in the construction) we shall take $M_{v}=S^{2}$.

Topology of the vertical space significantly changes the classification of lifts. Instead of $Z$ inequivalent regular homotopy sectors we obtain only two. The general argument presented previously says that lifts of one fold are classified by $\pi_{1}\left(S\left(S^{2}\right)\right)=Z_{2}$. The trivial element of this group corresponds to $I=0$, the non-trivial element to $I=1$. Now the cancellation of folds holds only for $\theta=\pi$, as for the particle case. In this way we fixed the value of the only free parameter in the model. It is in accordance with $\mathrm{YM}_{2}$ which for semi-simple Lie groups do not have any angle-like parameter.

\section{References}

[1] M. F. Atiyah and L. Jeffrey, Topological lagrangians and cohomology, J. Geom. Phys. 7 (1990) 119.

[2] W. A. Bardeen, I. Bars, A. J. Hanson and R. D. Peccei, Study of the longitudinal kink modes of the string, Phys. Rev. D 13 (1976), 2364.

[3] I. Bars, A quantum string theory of hadrons and its relation to quantum chromodynamics in two dimensions, Nuclear Phys. B 111 (1976), 1744.

[4] S. J. Blank and C. Curley, Desingularizing maps of corank one, Proc. Amer. Math. Soc. 80 (1980), 483.

[5] S. Corder, G. Moore and S. Ramgoolam, Large N $2 D$ Yang-Mills theory and topological string theory, Yale preprint YCTP-P23-93; hep-th/9402107. 
[6] W. Fulton, Hurwitz schemes and irreducibility of moduli of algebraic curves, Ann. of Math. (2) 90 (1969), 542.

[7] M. Golubitsky, V. Guillemin, Stable Mappings and Their Singularities, Springer, New York - Heidelberg, 1973.

[8] D. J. Gross, Two-dimensional QCD as a string theory, Nuclear Phys. B 400 (1993), 161; hep-th/9212149.

[9] D. J. Gross and W. Taylor, IV, Twists and loops in the string theory of two-dimensional QCD, Nuclear Phys. B 403 (1993), 395; hep-th/9303076.

[10] D. J. Gross and W. Taylor, IV, Two-dimensional QCD is a string theory, Nuclear Phys. B 400 (1993), 181; hep-th/9301068.

[11] J. Harris and D. Mumford, On the Kodaira dimension of the moduli space of curves, Invent. Math. 67 (1982), 23.

[12] W. Hirsch, Immersions of Manifolds, Trans. Amer. Math. Soc. 93 (1959), 242.

[13] G. 't Hooft, A planar diagram theory for strong interactions, Nuclear Phys. B 72 (1974), 461.

[14] G. 't Hooft, A two-dimensional model for mesons, Nuclear Phys. B 75 (1974), 461.

[15] P. Horava, Topological Rigid String Theory and Two Dimensional QCD, PUPT-1547, June 1995; hep-th/9507060.

[16] P. Horava, Topological Strings and QCD in Two Dimensions, to appear in: Proc. of The Cargese Workshop, 1993; hep-th/9311156.

[17] R. Lashof and S. Smale, On immersions of manifolds in Euclidean space, Ann. of Math. (2) 68 (1958), 562.

[18] V. Mathai and D. Quillen, Superconnections, Thom classes, and equivariant differential forms, Topology 25 (1986), 85.

[19] A. Migdal, Recursion equations in gauge field theories, Zh. Èksp. Teoret. Fiz. 69 (1975), 810; translated in Sov. Phys. JETP 42 (1975), 413.

[20] J. Pawełczy k, Immersions and folds in string theories of gauge fields, Internat. J. Modern Phys. A 11 (1996), 2661; hep-th/9604053.

[21] J. Pawełczyk, Two-dimensional string-theory model with no folds, Phys. Rev. Lett. 74 (1995), 3924; hep-th/9403175.

[22] B. Rusakov, Loop averages and partition function in $U(N)$ gauge theory on two-dimensional manifolds, Modern Phys. Lett. A 5 (1990), 693.

[23] S. Smale, Regular curves on Riemannian manifolds, Trans. Amer. Math. Soc. 87 (1958), 492.

[24] S. Smale, The classification of immersions of spheres in Euclidean spaces, Ann. of Math. (2) 69 (1959), 327.

[25] W. Thurston, The Geometry and Topology of Three-manifolds, Ch. 13, Princeton notes, 1977 (unpublished).

[26] H. Whitney, On singularities of maps of Euclidean spaces: $R^{2} \rightarrow R^{2}$ case, Ann. of Math. (2) 62 (1955), 374 .

[27] H. Whitney, The self-intersections of a smooth n-manifold in 2n-space, Ann. of Math. (2) 45 (1944), 220.

[28] E. Witten, Topological quantum field theory, Comm. Math. Phys. 117 (1988), 353. 